\title{
Mortality surveillance in the Netherlands: winter 2015/2016 of moderate severity
}

\author{
Liselotte van Asten ${ }^{\star 1}$, Marit de Lange', Anne Teirlinck', Frederika Dijkstra1, \\ Lenny Stoeldraijer ${ }^{2}$, Carel Harmsen ${ }^{2}$ and Wim van der Hoek ${ }^{1}$
}

${ }^{1}$ RIVM (Netherlands Institute of Public Health and the Environment, Centre for Infectious Disease Control, Bilthoven, Netherlands;

${ }^{2}$ Statistics Netherlands, Department of Demographic and socio-economic Statistics, The Hague, Netherlands

\section{Objective}

Weekly numbers of deaths are monitored to increase the capacity to deal with both expected and unusual (disease) events such as pandemic influenza, other infections and non-infectious incidents. The monitoring information can potentially be used to detect, track and estimate the impact of an outbreak or incident on all-cause mortality.

\section{Introduction}

The mortality monitoring system (initiated in 2009 during the influenza $\mathrm{A}(\mathrm{H} 1 \mathrm{~N} 1)$ pandemic) is a collaboration between the Centre for Infectious Disease Control (CIb) and Statistics Netherlands. The system monitors nation-wide reported number of deaths (population size 2014: 16.8 million) from all causes, as cause of death information is not available real-time. Data is received from Statistics Netherlands by weekly emails.

\section{Methods}

Once a week the number of reported deaths is checked for excess above expected levels at 2 different time-lags: within 1 and 2 weeks after date of death (covering a median $43 \%$ and $96 \%$ of all deaths respectively). A weekly email bulletin reporting the findings is sent to the Infectious Disease Early Warning Unit (at CIb) and a summary of results is posted on the RIVM website (National Institute for Public Health and the Environment). Any known concurrent and possibly related events are also reported. When excess deaths coincide with hot temperatures, the bulletin is sent to the Heat Plan Team (also at RIVM). Data are also sent to EuroMOMO which monitors excess mortality at a European level. For the Dutch system baselines and prediction limits are calculated using a 5 year historical period (updated each July). A serfling-like algorithm based on regression analysis is used to produce baselines which includes cyclical seasonal trends (models based on historical data in which weeks with extreme underreporting have been removed. Also periods with high excess mortality in winter and summer were removed so as not to influence the baseline with previous outbreaks).

\section{Results}

Increased mortality occurred during the entire influenza epidemic and up to three weeks thereafter (weeks 1-14 of 2016), except for a drop in week 7 (figure1). Excess mortality was primarily observed in persons 75 or older. Additionally, in several weeks mortality was increased in 65-74 year olds, (weeknr 4-6; peaking in week 4 with 564 deaths, when 468 baseline deaths were predicted). Also, in week 4 , mortality in the 25-34 year-old age group was significantly increased ( 25 deaths, while 14 were expected as baseline). Cumulative excess mortality was estimated at 3,900 deaths occurring during the 11 weeks of the 2015/2016 influenza epidemic and at 6,085 during the total winter season (44 weeks running from week 40 up to week 20).

\section{Conclusions}

In terms of number of deaths during the winter season (weeks 40-20) and during the influenza epidemic (weeks 1-11), the 2015/2016 season in the Netherlands was of moderate severity compared with the previous five years (and was of similar magnitude as the 2011/2012 winter). Notable was the short three-week time span with a higher peak in mortality in 65-74 year olds than has been observed in recent years. Although the influenza epidemic reached its peak in week 7 , the mortality data showed a dip in week 7 . The reason for the temporary decrease is unknown but there was a partial overlap with a public holiday.

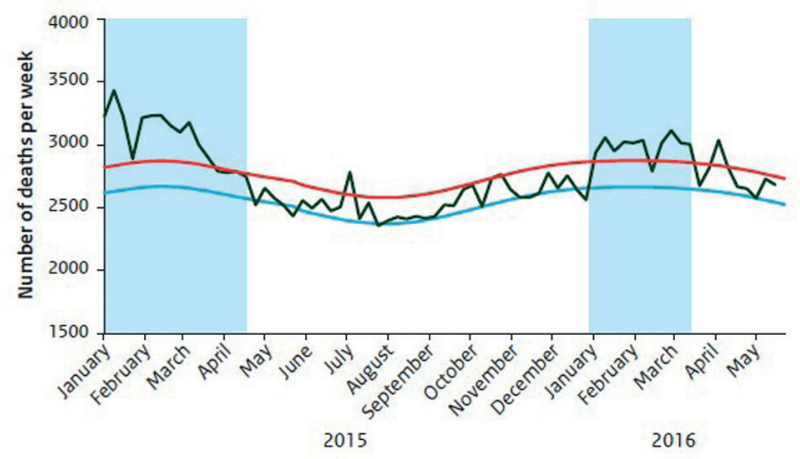

Figure 1. Weekly deaths reported within 2 weeks (black line) against baseline (blue) and prediction limit (red) for 2015-2016. Blue shading depicts influenza epidemic weeks (according to sentinel ILI surveillance).

\section{Keywords}

Mortality monitoring; influenza; Seasonality

\section{*Liselotte van Asten}

E-mail: liselotte.van.asten@rivm.nl 